\title{
latrogenic Perforation of Stomach - A Case Report
}

\author{
Nithin MD, Marigoudar RM, Rani S, Johnson LR
}

\author{
Department of Forensic Medicine and Toxicology \\ J.S.S University, J.S.S.Medical College. \\ Sri Shivarathreeshwara Nagar \\ Bannimantap, Mysore - 570015, \\ Karnataka, India. \\ Corresponding Author \\ M D Nithin \\ Department of Forensic Medicine and Toxicology \\ J.S.S University, J.S.S.Medical College. \\ Sri Shivarathreeshwara Nagar \\ Bannimantap, Mysore-570015, \\ Karnataka, India. \\ E-mail: nithin7755@gmail.com
}

\begin{abstract}
Gastric lavage is a routine procedure done in many cases of poisoning and it has been advocated by many as a lifesaving procedure. There may be some instances, where it might be unnecessary, ineffective or even detrimental to life. A 35 year old man walked into a casualty, 2 hours after having ingested 15 benzodiazepine tablets. Lavage was done by an unqualified person using Ewald's tube, leading to iatrogenic perforation. The unwarranted use of the procedure proved to be fatal.
\end{abstract}

\section{KEY WORDS}

Ewald's tube, gastric lavage, iatrogenic, unqualified person, unwarranted.

Citation

Jain MDN, Marigoudar RM, Rani S, Johnson RL. latrogenic Perforation of Stomach - A Case Report. Kathmandu Univ Med J 2015;50(2):175-7.

\section{INTRODUCTION}

Decontamination is the process of removing poison from the body. Various methods are employed in decontamination like inducing emesis, catharsis, administering activated charcoal, performing gastric lavage and whole bowel irrigation. ${ }^{1}$ Gastric lavage means washing out of the stomach often with water or a medicated solution. It has routinely been employed as part of the first line of management for the removal of unabsorbed ingested poisons. The apparatus used is the Ewald's tube, a non collapsible rubber tube measuring 1.5 meters in length and $1 \mathrm{~cm}$ in diameter, with a funnel, mouth gag and suction bulb. ${ }^{2}$ Here we present the case report of a patient who died while undergoing gastric lavage procedure being performed by an unqualified person.

\section{CASE REPORT}

A 35 year old man walked into a casualty, 2 hours after having ingested 15 benzodiazepine tablets. As part of routine management, it was decided to perform gastric lavage, which was performed by a ward boy using a Ewald's tube. As soon as the tube was inserted and the procedure was started, the patient developed seizures, collapsed and died on the table. Later a case of unnatural death was booked as it was thought that the death might have been due to poisoning.

\section{Autopsy findings}

The body was of an adult man, well built and wellnourished, measured $178 \mathrm{~cm}$ in length and weighed $107 \mathrm{~kg}$. Post mortem staining was present over the back and fixed. Rigor mortis was fully developed. External examination was unremarkable. On internal examination 3 litres of clear fluid was found in the peritoneal cavity (Figure 1). A perforation measuring $4 \mathrm{~cm} \times 3 \mathrm{~cm}$ was noticed in the middle of the lesser curvature of stomach. 
(Figure 2). All other internal organs were intact and congested.
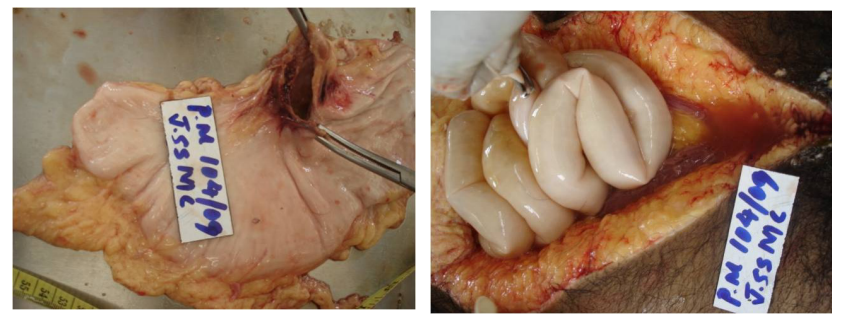

Figure 1. Perforation of Figure 2.3 litres of clear fluid in stomach measuring $4 \mathrm{~cm} \times 3 \mathrm{~cm}$ the peritoneal cavity

\section{DISCUSSION}

Gastric lavage has been employed for almost 2 centuries to remove poison from the stomach; however its usage has been associated with significant morbidities and in some cases, mortality. ${ }^{3}$ The incidence of complications due to gastric lavage is reported to be about $3 \%$, with the commonest being mucosal injury and bleeding. The less frequent complications include perforation of oesophagus or stomach, aspiration pneumonia, laryngeal spasm, fluid and electrolyte imbalance and vasovagal shock. This list is not exhaustive, and other unforeseen complications may occasionally occur. ${ }^{4}$

The significant risks associated with the use of Ewald's tube for gastric lavage has led to the recommendation that it should not be used for decontamination of ingested poisons. The "Lavacuator" is currently recommended as the ideal device for gastric decontamination as it has the following advantages over the Ewald's tube. ${ }^{5}$

1. Double lumen allows simultaneous lavage and evacuation of stomach.

2. Large elliptical eyes allow removal of even partially digested food particles.

3. Markings over the tube allow accurate assessment of the length of tube to be inserted.

4. Thermo-sensitive PVC reduces the risk of trauma to mucosal surface.

5. Moulded tip and eyes decreases incidence of trauma.

6. Flexible walls provide greater internal diameter.

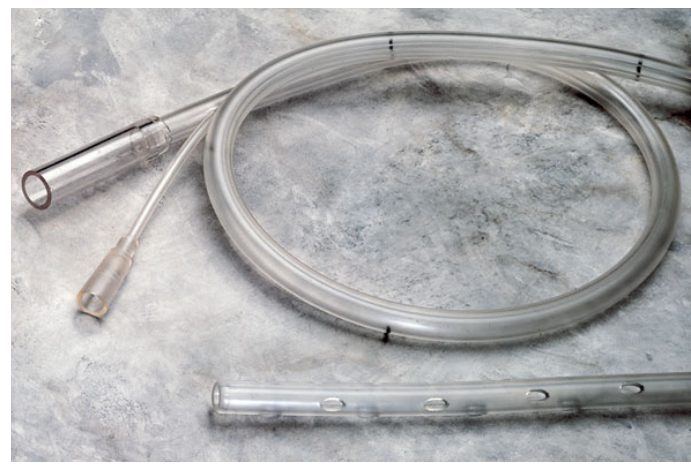

Figure 3. Lavacuator
Gastric lavage is a lifesaving procedure, but it is associated with significant risks, hence it should always be performed by trained personnel, under supervision and only when indicated. In this case the procedure was done by an unqualified, unskilled person, which led to death of the patient. A case was initially booked as that of unnatural death. Based on the autopsy findings, histopathology report the final opinion as to the cause of death was given as "peritonitis consequent upon perforation of lesser curvature of stomach". The police on the basis of the above report later converted the case into one of medical negligence, and booked it under Sec 304(A) IPC.

The question that is often raised is, who should perform the gastric lavage? The current health care system in India makes answering this question difficult.

There is an acute shortage of trained doctors working in the PHC's and the peripheries of the health network. It is these centres which see the majority of such cases. The lack of qualified personnel, forces the untrained staff to undertake such procedures without supervision. Nevertheless the casualty medical officer would be the appropriate qualified person for performing gastric lavage in the emergency room.

The casualty doctor also has the responsibility of deciding the indication for performing gastric lavage. Gastric lavage is not indicated for every case of poisoning. It is absolutely contra indicated in case of ingestion of corrosive poison, with the exception of carbolic acid. ${ }^{6}$

It is also not necessary to give a stomach wash for every case of overdose of tablets. The amount of tablets ingested, the effect of overdose of those tablets, the duration of time elapsed since ingestion of the tablets etc. are factors that should be considered before deciding to perform a gastric lavage.

The current recommendation for Gastric lavage is that it be should be performed only on patients who have ingested a life threatening dose of a drug/poison or who exhibit significant morbidity and present within 1-2 hours of ingestion.

"Do not perform gastric lavage on a person who has consumed a nontoxic substance or non toxic amount of toxic substance". ${ }^{7}$

\section{CONCLUSION}

It is prudent to take a full history where possible and quickly assess the clinical state of the patient prior to deciding to perform gastric lavage. The procedure should only be done by a qualified and trained person on a patient who warrants the procedure. Use the Lavacautor instead of Ewald's tube for gastric lavage.

The use of Ideal instrument in the hands of a qualified person could have prevented this death. 


\section{REFERENCES}

1. Pillay VV. General Management of Poisoning. In, Modern Medical Toxicology. $4^{\text {th }}$ ed. New Delhi: Jaypee Brothers Medical Publishers(P) Ltd; 2013:19-21.

2. Reddy KSN. General Considerations. In, The Essentials of Forensic Medicine and Toxicology. $30^{\text {th }}$ ed. Hyderabad: Sugunadevi K; 2011:470-471.

3. Benson BE, Hoppu K, Troutman WG, Bedry R, Erdman A, Höjer J, et al. Position Paper Update: Gastric Lavage for gastrointestinal decontamination. Clinical Toxicology 2013;51:140-6.
4. Gastric Lavage. Available: http://www21.ha.org.hk/files / PDF/Operations,\%20Tests\%20and\%20Procedures/A\&E_ GastricLavage_0183_eng.pdf.

5. http://www.soundveterinary.com.au/osc/product_info. php?products_id=923.

6. Vij K. Duties of a Doctor in cases of Suspected Poisoning.In, Textbook of Forensic Medicine and Toxicology, Principles and Practice. $4^{\text {th }}$ ed. New Delhi: Elsevier;2008:578-79.

7. General Management of Poisoning: www.revolutionpharmd.com 